\title{
CLOSED LOOP PERFORMANCE MONITORING IN THE PRESENCE OF SYSTEM CHANGES AND DISTURBANCES *
}

\author{
F. Gustafsson \\ Department of Electrical Engineering \\ Linköping University \\ S-581 83 Linköping, Sweden \\ fredrik@isy.liu.se
}

\author{
S.F Graebe \\ Department of Electrical and Computer Engineering \\ University of Newcastle \\ NSW 2308 Callaghan, Australia
}

Keywords: Change detection, adaptive control, stability margin, surveillance, disturbance rejection

\begin{abstract}
This paper addresses issues in closed loop performance monitoring. Particular attention is paid to detecting whether an observed deviation from nominal performance is due to a disturbance or due to a system change. This is achieved by introducing a novel performance measure that allows feasible application of a standard CUSUM change detector. The paper includes explicit results on the probability of mistaking a disturbance for a system change and demonstrates the algorithm with a simulation study.
\end{abstract}

\section{Introduction}

To motivate the following work, we briefly consider the wider topic of adaptive control. In conventional certainty equivalence adaptive control $[5,1]$, recursively estimated model parameters are used to recompute the controller parameters at every sampling instance. A difficulty with this strategy is that inevitable estimator transients, due to disturbances and parameter tracking, are passed to the controller.

It would therefore be preferable to update the controller only when the system has changed and the parameter estimates have converged with sufficient confidence. This can be achieved by viewing adaptive control as the coordination of the three subtasks of performance monitoring, estimation and control design. [4] gives an example of such a scheme as one of the contributions to the Sydney Benchmark [3].

With its application to supervised adaptive control in mind, the objective of this paper is to focus on the issue of closed loop performance monitoring.

\footnotetext{
* Work done whilst the first author was visiting the University of Newcastle. The first author gratefully acknowledges fundings from the Swedish Institute and the Swedish Natural Science Research Council during his visit.
}

At first sight, one might consider running a model of the nominal closed loop in parallel with the actual loop. This, however, is inadequate since disturbances and small model errors are not readily distinguished from control relevant system changes.

Alternatively, one might apply a standard fault detection scheme to detect system changes. Statistical tests as described, for instance in [2], are based on black-box models and could conceivably be applied to the current problem. This, however, is not exactly what is needed in the framework of adaptive control since there is no indication of whether a detected system change is control relevant or not. Furthermore, disturbances are very likely to cause false alarms. Another approach to fault detection is surveyed in [7]. Here the focus is on diagnosis and fault isolation. Although this is very control relevant information, the approach hinges on detailed prior knowledge of the system, for instance a physical model, which is not always available in adaptive control.

This paper describes a technique that utilizes statistical hypothesis testing to assess whether an observed deviation from nominal performance is due to a disturbance or due to a system change that has deteriorated the closed loop stability margins. To achieve this, the algorithm involves two key features. Firstly, there is an external probing signal. The associated perturbations in the output reflect the inherent trade-off between learning and control as known from the dual control principle [1]. Secondly, stability margins in the Nyquist plot are defined in terms of a novel clover like region rather than the conventional circle. The clover region maps to a linearly bounded region in the closed loop domain and therefore ensures feasible evaluation of the test statistics.

\section{Outline of the algorithm}

The following outline summarizes the algorithm and introduces notation. We will assume a system description in continuous time, although the derivation that follows is easily modified to discrete time. Consider the closed loop of Figure 1. Here $r(t)$ denotes the reference signal, $z(t)$ is the controlled output and $y(t)$ is the measured out- 


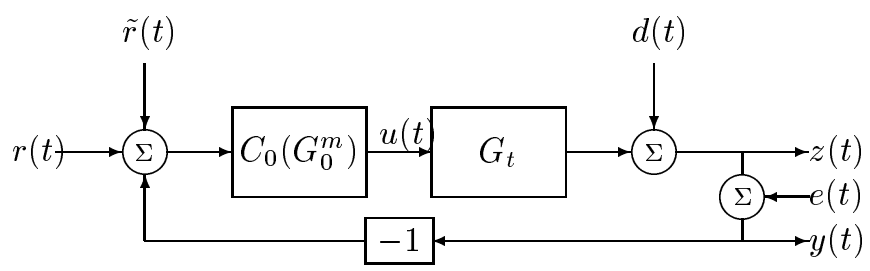

Figure 1: The closed loop system

put corrupted by the Brownian motion $e(t)$ with intensity $\sigma_{e}^{2} . d(t)$ represents an unmeasured deterministic disturbance and $\tilde{r}(t)$ is a perturbation signal discussed below. The true system at time $t$ is denoted $G_{t}$. At time $t=0$, when the true system was $G_{0}$, a model $G_{0}^{m}$ is assumed to be available for designing a controller $C_{0}$ achieving the nominal complementary sensitivity $T_{0}^{m}=\frac{C_{0} G_{0}^{m}}{1+C_{0} G_{0}^{m}}$. The true complementary sensitivity at time $t$ is then given by $T_{t}=\frac{C_{0} G_{t}}{1+C_{0} G_{t}}$ and the true sensitivity function is denoted $S_{t}=1-T_{t}$. The problem is to decide whether an observed difference

$$
\varepsilon(t)=y(t)-\hat{y}(t)=y(t)-T_{0}^{m} r(t)
$$

is due to a disturbance, or whether $G_{t}$ has changed in such a way that $T_{t}$ no longer posesses the prespecified stability margins and a new model should be estimated to design an adapted controller. We implicitely assume that the controller is not recomputed too often, in contrast to conventional adaptive control, so this extra feedback will not cause any stability problems. The test is carried out as follows.

1. $\tilde{r}(t)$, consisting of a sum of $M$ sinusoids of frequency $\omega_{k}$ and amplitude $\alpha_{k}$, is added to the reference signal to obtain excitation of the system,

$$
\begin{aligned}
& \bar{r}(t) \triangleq r(t)+\tilde{r}(t) \\
& \tilde{r}(t) \triangleq \sum_{k=1}^{M} \alpha_{k} \cos \omega_{k} t
\end{aligned}
$$

The test statistics $s_{k}(n)$ are computed as

$$
\begin{aligned}
s_{k}(n) \triangleq & T_{0}^{m}\left(\omega_{k}\right)+\frac{2}{\alpha_{k} T} \int_{n T-T}^{n T} \varepsilon(t) e^{i \omega_{k} t} d t \\
= & T_{0}^{m}\left(\omega_{k}\right)+\frac{2}{\alpha_{k} T} \int_{n T-T}^{n T}\left(\left(T_{t}-T_{0}^{m}\right)\right. \\
& \left.(r(t)+\tilde{r}(t))+S_{t}(d(t)+e(t))\right) e^{i \omega_{k} t} d t
\end{aligned}
$$

They are computed at the discrete times $n$ (for both discrete and continuous time systems) and for each frequency.

2. It can easily be shown that the test statistics can be written as

$$
s_{k}(n)=T_{t}\left(\omega_{k}\right)+D\left(n, \omega_{k}\right)+v_{k}(n),
$$
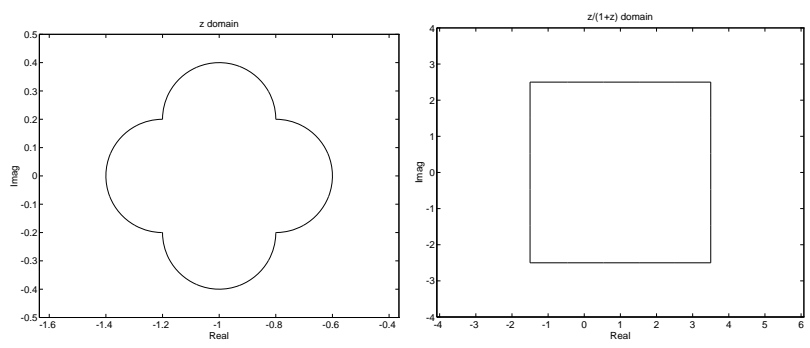

Figure 2: A clover (of radius $r=0.4$ ) in the $C G$ domain transforms to a rectangle in the $T$ domain.

where $D\left(n, \omega_{k}\right)$ is a deterministic (but unknown) term caused by the output disturbance which is defined in Section 4 and $v_{k}(n)$ is a noise term caused by the measurement noise $e(t)$, and it has variance $\operatorname{Var}\left(v_{k}(n)\right)=\frac{4 \sigma_{e}^{2}}{\alpha_{k}^{2} T}\left|S_{t}\left(\omega_{k}\right)\right|^{2}$.

3. Performance is specified for the Nyquist curve. The average of $s_{k}(n)$ is used for judging the performance in terms of the closed loop system. A new carefully designed performance criterion maps into a computationally convenient region in the closed loop domain, in which the average of $s_{k}(n)$ is expected to lie if the performance criterion is satisfied.

4. The influence of the term $D\left(n, \omega_{k}\right)$ is bounded in terms of the sensitivity function.

5. The well-known CUSUM test [6] is applied to $s_{k}(n)$.

\section{Performance criteria}

The question we would like to answer is if the timevariations of the true system $G_{t}$ have deteriorated the stability margin of the closed loop containing the timeinvariant controller $C_{0}$, designed for $G_{0}^{m}$.

We now introduce a novel performance/stability criterion, which can approximate the well-known phase and amplitude margin stability tests and the circle criterion. The advantage of the new criterion is that it maps into a linearly bounded region. This is exactly what we want because the test statistics $s_{k}(n)$ have known mean and variance and standard tests can be applied.

Definition 1 (Clover performance) An open-loop system has clover performance with radius $r$ if its Nyquist curve is outside the clover depicted in Figure 2, where each circle has radius $r / 2$.

The following lemma motivates the clover region.

Lemma 1 The Möbius transformation $\frac{z}{z+1}$ transforms the clover into a rectangle alligned to the real and imaginary axis passing the points $1+\frac{1}{r}, 1-\frac{1}{r}, 1+\frac{i}{r}$ and $1-\frac{i}{r}$ respectively. That is, the borders are passing the real and imaginary axis at $\nu_{1}=1+\frac{1}{r}, \nu_{2}=1-\frac{1}{r}, \nu_{3}=\frac{1}{r}$ and $\nu_{4}=-\frac{1}{r}$, respectively. 
Proof: All circles pass the point -1 which maps to infinity, so the images are four straight lines. The four points given in the Lemma are the images of the circles for $\omega$ equal to $0, \pi, \pi / 2$ and $-\pi / 2$ respectively. Finally, the intersections of the four circles form right angles and so do the four lines since $z /(z+1)$ is a conformal mapping.

\section{Influence of the disturbance}

The influence of the output step disturbance on the test statistics is precisely quantified in the following theorem. When considering disturbances, we may assume a timeinvariant system. The effect of a step disturbance for instance lasts as long as the impulse response of the sensitivity function $S_{t}=1-T_{t}$. If the time-variations of the sensitivity function are fast compared to its dynamics it is not at all clear what is meant by a "linear system". Thus, we can assume that the sensitivity function is time invariant during a disturbance.

Theorem 1 The disturbance term $D\left(n, \omega_{k}\right)$ in (5) is bounded as follows:

$$
\begin{aligned}
\left|D\left(n, \omega_{k}\right)\right| & \leq \frac{4 d_{A}}{\alpha_{k} \omega_{k}} \max _{0 \leq t<T}\left|s\left(t+n T-t_{0}\right)\right| \\
\left|\sum_{n=0}^{\infty} D\left(n, \omega_{k}\right)\right| & \leq \frac{2 d_{A}}{\alpha_{k} \omega_{k} T}\left|S\left(\omega_{k}\right)-S(0)\right|
\end{aligned}
$$

Here $s(t)$ is the impulse response of the time invariant sensitivity function $S(\omega)$.

Proof: The influence of the step $d_{A} \sigma\left(t-t_{0}\right)$ on the output $y(t)$ is

$$
S d_{A} \sigma\left(t-t_{0}\right)=\int_{-\infty}^{\infty} s(\tau) A \sigma\left(t-\tau-t_{0}\right) d \tau
$$

From (3) we have

$$
D\left(n, \omega_{k}\right) \triangleq \frac{2}{\alpha_{k} T} \int_{n T-T}^{n T} S_{t} d(t) e^{i \omega_{k} t} d t
$$

This gives

$$
\begin{aligned}
& D\left(n, \omega_{k}\right)= \\
= & \frac{2}{\alpha_{k} T} \int_{n T-T}^{n T} S d(t) e^{i \omega_{k} t} d t \\
= & \frac{2}{\alpha_{k} T} \int_{n T-T}^{n T} \int_{-\infty}^{\infty} s(\tau) d_{A} \sigma\left(t-\tau-t_{0}\right) d \tau e^{j \omega_{k} t} d t \\
= & \frac{2 d_{A}}{\alpha_{k} T} \int_{-\infty}^{\infty} s(\tau) \int_{n T-T}^{n T} \sigma\left(t-\tau-t_{0}\right) e^{j \omega_{k} t} d t d \tau \\
= & \frac{2 d_{A}}{\alpha_{k} T} \int_{-\infty}^{\infty} s(\tau) \int_{\min \left(n T-T, \tau+t_{0}\right)}^{n T} e^{j \omega_{k} t} d t d \tau \\
= & \frac{2 d_{A}}{\alpha_{k} T} \int_{-\infty}^{n T-T-t_{0}} s(\tau) \frac{1}{j \omega_{k}}\left(e^{j \omega_{k} n T}-e^{j \omega_{k}(n T-T)}\right) d \tau \\
+ & \frac{2 d_{A}}{\alpha_{k} T} \int_{n T-T-t_{0}}^{n T-t_{0}} s(\tau) \frac{1}{j \omega_{k}}\left(e^{j \omega_{k} n T}-e^{j \omega_{k}\left(\tau+t_{0}\right)}\right) d \tau \\
= & \frac{2 d_{A}}{\alpha_{k} T} \frac{j}{\omega_{k}} \int_{0}^{T} s\left(\tau+n T-T-t_{0}\right)\left(e^{j \omega_{k} \tau}-1\right) d \tau
\end{aligned}
$$

and (6) follows. Time invariance is used in the second and the last equalities, where it is also used that the integration interval $T$ is matched to $\omega_{k}$ such that $e^{j \omega_{k} n T}=1$. We had to distinguish two different cases, one where $t_{0}+\tau \in[n T-T, n T]$ and one where it is not. Summing up over $n$ now gives

$$
\begin{aligned}
\sum_{n} D\left(n, \omega_{k}\right) & =\frac{j 2 d_{A}}{\alpha \omega_{k} T} \int_{0}^{\infty} s(\tau)\left(e^{j \omega_{k} \tau}-1\right) d \tau \\
& =\frac{j 2 d_{A}}{\alpha_{k} \omega_{k} T}\left(S\left(\omega_{k}\right)-S(0)\right)
\end{aligned}
$$

and (7) follows.

Since $S(s)$ is a stable transfer function, its impulse response decays exponentially. Thus, (6) converges to zero exponentially.

\section{Applying the CUSUM test}

In this section a review of the CUSUM test is given first and then it is applied to the problem at hand.

Consider the case of an unknown constant in white Gaussian noise:

$$
\begin{aligned}
& s(n)=\theta+\sigma e(n) \\
& e(n) \in \mathrm{N}(0,1)
\end{aligned}
$$

We want to detect a significant increase in the mean $\theta$ :

$$
\begin{array}{ll}
H_{0}: & \theta<\nu \\
H_{1}: & \theta>\nu .
\end{array}
$$

The CUSUM test, see [6] or [2] page 41 ff, applies and it is given by

$$
\begin{aligned}
t_{a} & =\min \{n: g(n) \geq h\} \\
g(n) & =\max (0, g(n-1)+s(n)-\nu)
\end{aligned}
$$


The average run length (ARL) function is defined as

$$
\tau_{\theta}=E\left(t_{a} \mid \theta\right)=A R L(h, \nu, \sigma, \theta),
$$

and it is a function of $h, \nu, \sigma$ and $\theta$. Basically, we want $\tau_{\theta}$ to be large under $H_{0}$ and small under $H_{1}$. The mean time between false alarms is defined as $\tau_{0}$ and the mean time for detection as $\tau_{\nu}$.

An accurate explicit approximation is derived in [8], which for small $\frac{\nu-\theta}{\sigma}<0$ yields

$$
\tau_{\theta}=\frac{h+1.166}{\theta-\nu} .
$$

This expression can be used to compute the threshold $h$ from a desired delay for detection.

In our problem, we want to test the following hypotheses:

$$
\begin{array}{ll}
H_{0}: & T_{t}(\omega) \text { is inside a rectangle for all } \omega \\
H_{1}: & T_{t}(\omega) \text { is outside a rectangle for some } \omega .
\end{array}
$$

That is, the test must detect four different events; the mean of the real and imaginary parts, respectively must not be larger or smaller than some constants. Assume for a while that there is no disturbance, so $s_{k}(n)=T_{t}\left(\omega_{k}\right)+$ $v_{k}(n)$. Here we identify $\theta$ in (9) by $\operatorname{Re} T_{0},-\operatorname{Re} T_{0}, \operatorname{Im} T_{0}$ and $-\operatorname{Im} T_{0}$, respectively. From (3) and Lemma 1, four CUSUM test can be applied directly. The full test is

$t_{a}=\min \left\{n: g_{k}^{i}(n) \geq h\right.$ any $\left.k=1, . ., M, i=1: 4\right\}$

$g_{k}^{1}(n)=\max \left(0, g_{k}^{1}(n-1)+\operatorname{Re} s_{k}(n)-\nu^{1}\right), k=1: M$

$g_{k}^{2}(n)=\max \left(0, g_{k}^{2}(n-1)-\operatorname{Re} s_{k}(n)-\nu^{2}\right), k=1: M$

$g_{k}^{3}(n)=\max \left(0, g_{k}^{3}(n-1)+\operatorname{Im} s_{k}(n)-\nu^{3}\right), k=1: M$

$g_{k}^{4}(n)=\max \left(0, g_{k}^{4}(n-1)-\operatorname{Im} s_{k}(n)-\nu^{4}\right), k=1: M$

where $i$ indexes the four sides of the rectangle, $k$ indexes the frequency, $t_{a}$ denotes the time of an alarm and $h$ is a threshold that influences the stochastic properties of the test, usually expressed in false alarm rate and delay for detection. The convention is that each $g_{k}(n)$ is set to zero initially and after each alarm. The idea is to continuously monitor the quantity $\max _{i, k} g_{k}^{i}(n)$. If is exceeds the threshold $h$, the algorithm signals for a decrease in stability margin.

\section{Choosing the design parameters}

An advantage with the described test is that the design can be automated. Below, a brief overview of the design procedure is given. The following is assumed to be given:

1. Specification of $r_{1}$ in the performance criterion and a $r_{0}<r_{1}$ for defining mean time to detection.

2. An initial model $G^{m}$ of the plant $G_{0}$ and a controller $C_{0}$ satisfying $\left|1+C_{0} G_{m}\right|>r_{1}$.

3. The mean time for detection $\tau_{1}$.
4. A bound on how large the perturbation signal is allowed to be at the output. A guideline is to accept the same output degradation as the measurement noise causes.

5. A set of critical frequencies $\left\{\omega_{k}\right\}$ or prior knowledge of possible changes which can be condensed to the constants $\omega_{l}, \omega_{h}, C_{3}$ and $\gamma$ in

$$
\begin{array}{ccc}
\left|C_{0}(\omega) G_{t}(\omega)\right|>1+r_{1}, & \omega<\omega_{l} & (14) \\
\left|C_{0}(\omega) G_{t}(\omega)\right|<1-r_{1}, & \omega>\omega_{h} & (15) \\
\left|C_{0}\left(\omega_{1}\right) G_{t}\left(\omega_{1}\right)-C_{0}\left(\omega_{2}\right) G_{t}\left(\omega_{2}\right)\right| & & \\
<C_{3}\left|\omega_{1}-\omega_{2}\right|^{\gamma}, & \omega_{l}<\omega<\omega_{h}
\end{array}
$$

It can be shown that these inequalities can be used to compute the critical frequencies. From (14) and (15) a critical frequency interval is obtained. Inequality (16) tells us what how much the Nyquist curve might change between two frequencies, and this gives the necessary frequency separation.

Note that the above specifications are commonly assumed to be known in control design, except for the mean time for detection. Inequalities (14) and (15) are given from standard frequency uncertainty specifications and inequality (16) is a Lipschitz continuity assumption.

Now, the amplitudes on the test sinusoids can be computed from 3, for instance through $\alpha_{k}=\frac{S^{m}\left(\omega_{k}\right)}{T^{m}\left(\omega_{k}\right)} \sigma_{e}$. The integration time is given by $T=2 \pi / \min _{k}\left(\omega_{k}\right)$. The mean time for detection can be shown to give the CUSUM threshold using (12) as $h=\frac{\tau_{1}}{T} \frac{r_{1}-r_{0}}{r_{0} r_{1}}$. Finally, we can compute the variance of the test statistics as $\sigma_{s, k}^{2}=\left|S^{m}\left(\omega_{k}\right)\right|^{2} \frac{4 \sigma_{e}^{2}}{\alpha_{k}^{2} T}$.

It remains to check if the mean time between alarms and robustness to step disturbances are satisfactory:

$$
\begin{aligned}
\tau_{0} & =f\left(\frac{h}{\sigma_{s, k}}, \frac{\min _{i}\left(\nu_{i}-\theta_{k}^{i}\right)}{\sigma_{s, k}}\right) \\
d_{A} & <\frac{\alpha_{k} \omega_{k} h T}{2\left|S_{t}\left(\omega_{k}\right)-S_{t}(0)\right|} \\
d_{A} & <\frac{\alpha_{k} \omega_{k} h}{4 \max _{t}|s(t)|}
\end{aligned}
$$

If this is not the case, we may try to increase the perturbation amplitudes or accept a longer delay for detection.

We will illustrate the design process with an example.

\section{$7 \quad$ A simulated example}

The performance detector is here applied to a simulated DC motor with transfer function

$$
G_{0}(s)=\frac{b}{s(s+a)}, \quad b=1, a=2 ;
$$

The measurement noise has intensity $0.01^{2}$. A proportional controller with $C_{p}=10$ is chosen, which gives 
high bandwidth and reasonably damped step response. The controller satisfies the clover criterion with radius $r_{1}=0.6$. This is the nominal performance and the mean time to detection is computed for a clover performance with radius 0.3 . Figure 3 shows the nominal Nyquist curve, the clovers and their Möbius transforms.

Suppose we have prior knowledge of possible changes in $a$ in the interval $[1,2.2]$. This can be converted to the inequalities (14) to (16) and leads to that four critical frequencies $\omega=2,3.25,4.5,5.75$ of the Nyquist curve need to be examined. Choosing the test amplitudes to 0.02 implies that the sinusoids amplitudes at the output is in the same order as the output noise. Thus, the reference signal is

$$
\begin{aligned}
\bar{r}(t)= & r(t)+0.02 \cos (2 t)+0.02 \cos (3.25 t) \\
& +0.02 \cos (4.5 t)+0.02 \cos (5.75 t)
\end{aligned}
$$

The integration time is chosen to $T=3.14$ so the integral in (3) is taken over a multiple of the period times of the test frequencies. The delay for detection is chosen to 15 seconds.

The design procedure leads to the following: The threshold in the CUSUM test is $h=8$. The properties of the test are summarized in the table below.

\begin{tabular}{||l||c|c|c|c||}
\hline Property & $\mathrm{k}=1$ & $\mathrm{k}=2$ & $\mathrm{k}=3$ & $\mathrm{k}=4$ \\
\hline \hline Frequency & 2 & 3.25 & 4.5 & 5.75 \\
\hline Amplitude & 0.02 & 0.02 & 0.02 & 0.02 \\
\hline Sinusoids' amplitude at output & 0.03 & 0.03 & 0.01 & 0.01 \\
\hline Signal to noise ratio at output & 3.5 & 1.6 & 0.9 & 0.8 \\
\hline Std $\left(s_{k}(n)\right)$ & 0.44 & 1.1 & 0.92 & 0.77 \\
\hline max step amplitude & 0.6 & 0.4 & 0.7 & 1 \\
\hline Mean time between false alarm & $9 \mathrm{~T}$ & $39 \mathrm{~T}$ & $49 \mathrm{~T}$ & $25 \mathrm{~T}$ \\
\hline
\end{tabular}

Figure 4 shows a simulation. The reference signal changes from 0 to 1 at time 16 and back to 0 at time 123. At time $t=28$ a step disturbance of amplitude -0.2 , which is well below the maximum amplitude 0.4 given in the table, is added to the output and removed at time $t=44$. The value of $a$ changes from 2 to 1 at $t=60$. As seen, the performance of tracking reference changes deteriorates after the system change.

The upper plot of Figure 4 shows $\max \left(g_{k}(t)\right)$. We get a first warning already at time 63 . If we had examined the functions $g_{k}(t)$ individually, we would have found that the lower leaf causes two alarms for frequency 3.25 at times $63,79,97$ and 126, while the right leaf causes alarms at times 97 and 132 . Note that the mean time between alarms is roughly 15 seconds.

This extra information can for instance be used to modify the controller by including a lead filter designed to have a phase advance at $\omega=3.25$. This is an immediate action that can be undertaken before a new accurate model of the system is identified and a new controller is computed.

The step disturbance gives two peaks when it enters and disappears, but they are well below the threshold as
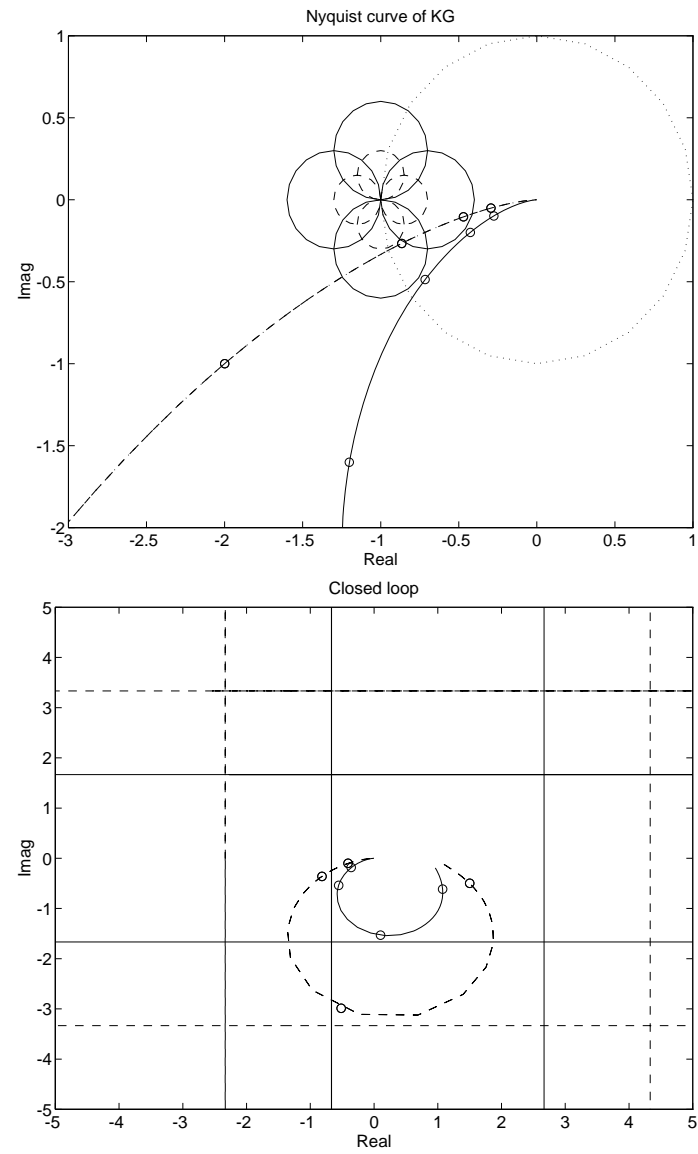

Figure 3: The left plot shows the Nyquist curve for the nominal plant (solid) and the plant after a pole change (dashed). The circles are located at the frequencies under consideration. Also shown is the clover criterion (solid) and the clover used to compute mean time to detection (dashed) and the unit circle (dotted). The right plot shows the same thing in the closed loop domain.

predicted in the design. It is also interesting to note that a disturbance and a system change give rise to the same type of response of the closed loop output, though the influence of the system change happens to be somewhat larger in this particular simulation. Thus, it is almost impossible to distinguish these two kinds of "faults" from the output and a constant reference signal alone.

\section{Conclusions}

In this paper we have drawn attention to particular requirements for performance monitoring in the larger context of supervised adaptive control. In particular, we have highlighted the need to detect control relevant system changes in closed loop operation and to distinguish them from disturbances. To this end, we replaced the more conventional circular stability margins as known from the small gain theorem by a novel clover like region. This criterion had the advantage of mapping into a 

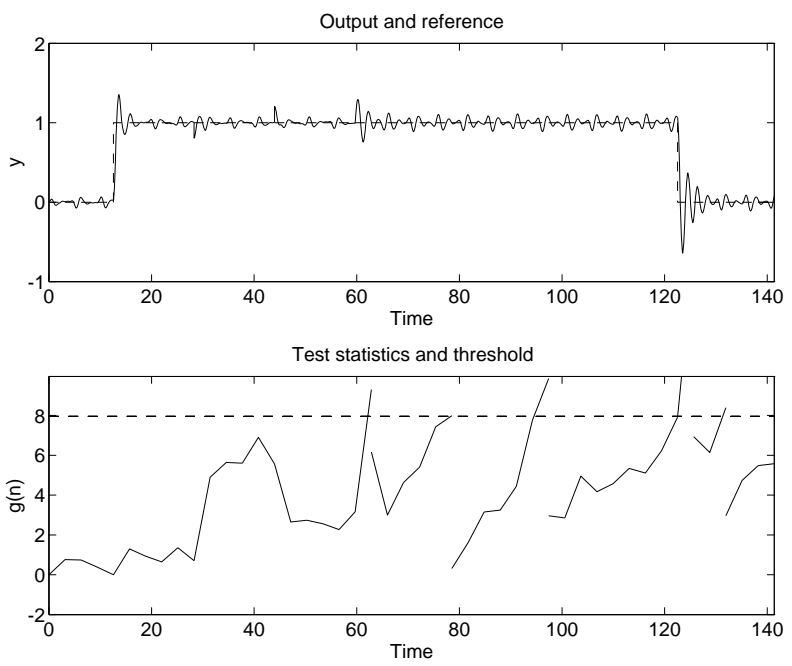

Figure 4: The upper plot shows the (noise free) output and the reference signal, the lower plot shows the test statistics and the threshold.

rectangular region in the closed loop domain, while still approximating the conventional criteria of phase margin, amplitude margin and small gain theorem.

The rectangular region allows feasible evaluation of statistical hypotheses testing. As a consequence we were able to derive explicit expressions for the probability mistaking a disturbance for a system change and give physically insightful guidelines for the tuning parameters. The necessity of a small external probing signal is in accordance with the well-known duality between learning and control.

\section{References}

[1] K.J. Åstrom and B. Wittenmark. Adaptive Control. Addison Wesley, 1989.

[2] M. Basseville and I.V. Nikiforov. Detection of abrupt changes: theory and application. Information and system science series. Prentice Hall, Englewood Cliffs, NJ., 1993.

[3] S. F. Graebe. Robust and adaptive control of an unknown plant: A benchmark of a new format. Automatica, 30(4):567-575, 1994.

[4] O. Hecker, T. Knapp, and R. Isermann. Robust adaptive control of a time varying process using parallel recursive estimators. Automatica, 30:599-604, 1994.

[5] R.H. Middleton and G.C. Goodwin. Digital Estimation and Control: A Unified Approach. Prentice-Hall, Inc., New Jersey, 1990.

[6] E.S Page. Continuous inspection schemes. Biometrika, 41:100-115, 1954.
[7] R. Patton, P. Frank, and R. Clark. Fault diagnosis in dynamic systems. Prentice Hall, 1989.

[8] D. Siegmund. Sequential analysis - tests and confidence intervals. Series in statistics. Springer, 1985. 